

\title{
Diversidad estructural y semiótica en el proceso didáctico de ampliación de los naturales a los enteros: un estudio sobre comprensión en el campo de la relatividad aditiva
}

\author{
José Luis González Marí \\ Luis Rico Romero \\ Jesús Gallardo Romero
}

Departamento de Didactica de la Matemática, de las Ciencias Sociales y de las Ciencias Experimentales. Universidad de Málaga

\section{España}

José Luis González Marí. Departamento de Didactica de la Matemática, de las Ciencias Sociales y de las Ciencias Experimentales. Facultad de Ciencias de la Educación. Universidad de Málaga. Campus de Teatinos s/nº 29071 Mälaga. España. E-mail: gmari@uma.es

(C) Education \& Psychology I+D+i and Editorial EOS (Spain) 


\section{Resumen}

Introducción. En el proceso didáctico de ampliación de los naturales a los enteros interviene un tipo de medidas cuya integración en los sistemas numéricos conocidos - naturales y enteros - no es inmediata ni correcta. Por el contrario, se ajustan a una tercera estructura numérica - sistema de los números naturales relativos - que presenta cinco diferencias con respecto a la estructura de los números enteros. En este trabajo se expone una parte del estudio realizado para comprobar que dichas diferencias también se manifiestan en el ámbito cognitivo.

Método. Se emplea el metaanálisis cualitativo y el análisis didáctico para el estudio teórico y el análisis de respuestas, estudio descriptivo y análisis de correspondencias a respuestas a cuestionarios estructurados.

Resultados. Las estructuras algebraicas y la naturaleza epistemologica y fenomenológica de los números naturales relativos y los números enteros son diferentes, y los sujetos distinguen ambos tipos de números respondiendo de manera diferente a situaciones propias de ambas estructuras numéricas.

Discusión. Los resultados confirman las conjeturas y refuerzan la necesidad didáctica de una nueva organización para el campo aditivo elemental en la que se tenga en cuenta la relatividad aditiva y los números naturales relativos, al igual que se hace con la proporcionalidad y los números fraccionarios en el campo multiplicativo.

Palabras clave: Enteros, Numerous dirigidos, Campo conceptual aditivo, Pensamiento numérico, Comprensión de la aritmética.

Recibido: 19/12/08 Aceptación inicial: 30/12/08 Aceptación final: 23/01/09 


\begin{abstract}
Introduction. In the current didactical process dealing with the transition from natural numbers to integers, is working a kind of measurements with no inmediate, neither correct from a conceptual point of view, integration within the known numerical systems. We refer to metric and comparative notions for which a third numerical system - that we now call "relative natural" numbers - is needed; it is a numerical system which presents five structural differences from those of natural numbers and integers. In this paper we bring to front a part of a study fulfilled to make evident that the above mentioned differences are also cognitive.
\end{abstract}

Method. Qualitative metaanalysis and Didactical Analysis for theoretical questions and descriptive statistics and correspondences analysis to structurated questionnaires responses for the empirical study.

Results. The epistemology and phenomenology of relative natural nusmber and integer numbers systems are different and a sample of individual responses to a structured questionnary show diferenciated meanings and internal representations when they answer to questions about the two kinds of situations from both numerical structures.

Discussion. The results are agree with conjectures and claim for a new organization for the additive numerical field, in which additive relativity and relative natural numbers would be considered, as it is the case of proportionality and fractions in the multiplicative conceptual field.

Key words: Integers; Directed numbers; Additive conceptual field; Numerical thinking; Understanding arithmetic.

Received: 12/19/08 Initial Acceptance: 12/30/08 Final Acceptance: 01/23/09

\title{
Introducción
}

Durante más de tres milenios de Historia de la Matemática (Gericke, 1996), en lo que se ha llamado "un proceso histórico sorprendente" (Glaeser, 1981), han coexistido, de manera informal (Clawson, 1994), diferentes tipos de conceptos numéricos en el campo aditivo (Schubring, 1986). De entre ellos, los que hoy conocemos como enteros negativos y positivos 
(Euler, 1840), son resultado de un proceso de formalización culminado con la aplicación del principio de permanencia de las leyes formales (Pycior, 1981). Dicho proceso, parcialmente justificado desde el punto de vista lógico (Russell, 1973) ${ }^{1}$ y bien establecido desde el punto de vista matemático (Feferman, 1989), no soluciona, sin embargo, numerosos problemas que surgen en la Educación Matemática (González y otros, 1990). El motivo fundamental radica en la existencia, en el campo de los fenómenos de aplicación de las estructuras aditivas y ordinales de los números naturales y los números enteros, de un tipo de situaciones, a las que llamamos "situaciones relativas aditivas", en las que intervienen unas medidas cuya naturaleza y funcionamiento se ajustan a una estructura numérica no usual a la que hemos denominado sistema de los "números naturales relativos". Se trata de nociones propias del campo conceptual aditivo que han estado informalmente presentes a lo largo del desarrollo histórico de los números negativos (Glaeser, 1981; González y otros, 1990) y que han sido eclipsadas por la construcción formal de los números enteros (González, 1995, pp. 195 - 232) ? $^{3}$

El problema que abordamos se sitúa en un hecho comprobable (Maz, 2000): la estructura numérica mediante la que algunos matemáticos anteriores al siglo XIX, e incluso con posterioridad, inician la presentación del conjunto de los enteros no es la de grupo aditivo totalmente ordenado. Las situaciones elegidas hacen intervenir medidas y relaciones que no satisfacen las condiciones requeridas para ejemplificar tales números. Veamos un ejemplo:

"Esta señal + se llama positiva; y esta - negativa. De aquí nace que siempre que una cantidad tiene delante de sí la señal +, o no tiene delante de si la señal -, se llama cantidad positiva; pero siempre que una cantidad tiene delante de sí la señal se llama cantidad negativa, y cuando a tal cantidad no antecede otra positiva esta cantidad se llama cantidad falsa o menor que nada. Es fácil formar ideas de estas cantidades menores que nada. Supongase que desde C hasta B hay tres leguas y desde C hasta A hay dos.

\footnotetext{
${ }^{1}$ El autor, a partir de un análisis lógico del orden, atribuye la diferencia de sentido y la diferencia de signo a la relación genérica existente entre una relación asimétrica y su recíproca, identificando los enteros negativos y positivos con las sucesivas potencias de ambas relaciones. Decimos que la justificación es parcial porque creemos que los argumentos de Russell inciden sobre la existencia de los números naturales relativos y no de la de los enteros.

${ }^{2}$ Aquellas que aparecen en problemas aritméticos en los que intervienen cantidades o medidas discretas elementales que operan entre sí bajo uno de los tres tipos de relaciones aditivas - combinación, cambio o comparación y de las que una de ellas, al menos, es una cantidad/medida natural relativa. Cantidades naturales relativas son cantidades naturales adjetivadas dualmente (positivas / negativas, más / menos) (González, 1995, pp. 261-263).

${ }^{3}$ Tesis Doctoral leída en el Departamento de Didáctica de la Matemática de la Universidad de Granada.
} 


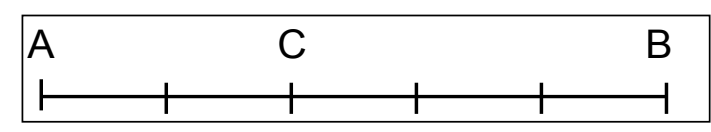

Figura 0. Ilustración del texto de Ulloa

Si un caminante está en $C$ con designio de llegar a $B$ y efectivamente saliendo de Cllega a B, es verdad decir que avanzó y que su avance es mayor que nada y que la medida de este avance es tres leguas. Si no obstante su designio se halla precisado a detenerle en $C$ su avance es ninguno, o es igual a nada. Si en vez de caminar hacia $B$ saliendo de C, llega a A, en la frase vulgar dirá que cejó, y para expresar que hizo lo contrario de lo que había de hacer puede decirse que ha avanzado menos que nada y que su avance es -2 leguas, conque aqui dos leguas son menos que nada." (Ulloa, 1706; pp. 20-22).

El ejemplo elegido no responde a las condiciones formales de la estructura de grupo aditivo totalmente ordenado de los números enteros. En primer lugar, el modelo utilizado implica la existencia de dos conjuntos totalmente ordenados, cada uno con primer elemento: el correspondiente a las cantidades/medidas tomadas como "avances positivos", que comienzan en un "avance ninguno", y el de las cantidades/medidas tomadas como retrocesos o "avances negativos", a los que el autor caracteriza como "avanzar menos que nada”, que deben comenzar por un "retroceso ninguno"; surge, por tanto, la necesidad lógica de considerar dos ceros en lugar de uno sólo.

Por otra parte, si bien el autor no lo menciona, el uso común establece que retroceder o cejar 7 leguas es más que retroceder sólo 3 leguas, lo que se ajusta a una relación de orden inversa a la usual entre los números enteros negativos e indica que en cada uno de los dos subconjuntos ("avances" y "retrocesos") se mantiene el mismo orden natural. Igualmente, comparar un avance específico con un retroceso específico resulta forzado y arbitrario, ya que, por un lado, se trata de dos tipos distintos de acciones y de cantidades/medidas ${ }^{4} \mathrm{y}$, por otro, son igualmente válidos cualesquiera de los dos sentidos que se pueden adoptar para el orden, lo que apunta hacia la independencia de los dos subconjuntos, a diferencia de lo que ocurre en la estructura entera. Por tanto, la estructura que se ejemplifica no es la de un orden

\footnotetext{
${ }^{4}$ Conocidas como "dirigidas" o "adjetivadas" (Bell, 1982).
} 
total sin primer ni último elementos, sino la de un orden parcial sobre dos subconjuntos disjuntos (el de las cantidades/medidas "positivas" y el de las "negativas" ), cada uno totalmente ordenado y con primer elemento ("avanzar nada" y "retroceder nada", respectivamente) (González, 1995; pp. 224). En la figura 1 se representan sendos diagramas que ilustran el orden existente y la situación y características de los ceros en las tres estructuras numéricas.

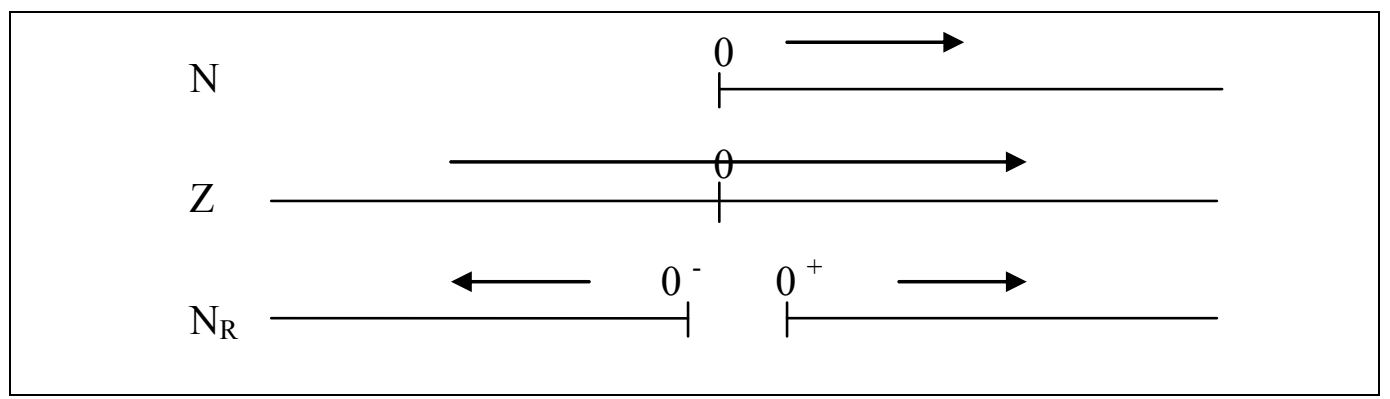

Figura 1. Una representación de los tres tipos de números

Por otra parte, la estructura operatoria de los números presentados en el ejemplo tampoco es la de grupo aditivo. El análisis detallado permite identificar una estructura de semigrupo aditivo no conmutativo, sin elemento neutro y con dos elementos nulos no permutables entre sí, con las operaciones usuales de suma y resta entre números naturales para valores del mismo "signo" y una ley basada en unas reglas de "anulación-compensación"6 para valores de signos opuestos. En consecuencia, podemos afirmar que el conjunto que se ejemplifica (o se estudia) es el de los números naturales relativos y no el de los enteros. La confusión se agrava cuando, además, se mezclan elementos de ambas estructuras y, sobre todo, ante la ausencia de una simbolización específica para los números naturales relativos, se utiliza la notación entera para los dos tipos de números, lo que conduce a su identificación con las consiguientes disfuncionalidades.

El problema planteado admite distintos enfoques (epistemológico, histórico o matemático, por ejemplo), pero no es nuestra intención entrar en otras consideraciones que no sean las exclusivamente didácticas. Nuestro interés se centra, por tanto, en el ámbito de los fenómeno que se originan cuando se trata de transmitir, enseñar, explicar o "hacer comprender" nociones matemáticas formales mediante el clásico recurso didáctico a los ejemplos concretos, intuitivos y familiares; un recurso necesario y adecuado en muchos casos pero problemá-

\footnotetext{
${ }^{5}$ Las comillas indican que estos términos no se emplean aquí con los mismos significados que en el caso de los números con signo, sino sólo para calificar la dualidad propia de la estructura natural relativa.
} 
tico en otros, en los que los propios ejemplos producen desajustes, incluso estructurales, que es necesario identificar y tratar adecuadamente. En tal sentido, hemos desarrollado una investigación orientada a identificar, analizar y organizar, a partir de consideraciones epistemológicas, cognitivas, fenomenológicas y didácticas, la parte del campo conceptual aditivo (Vergnaud, 1993, pp. 97 y sgtes.) a la que hemos denominado "campo conceptual de los números naturales relativos".

En el presente artículo se expone una parte del estudio empírico realizado para poner de manifiesto que las diferencias entre las distintas nociones métricas y numéricas que intervienen no sólo son estructurales o fenomenológicas, sino que tambien son cognitivas, es decir, que los sujetos manejan y asignan significados diferentes a situaciones usualmente consideradas del mismo tipo y tratadas por igual mediante los números enteros. Esta diferenciación incide en la existencia y necesidad didáctica del nuevo campo conceptual, cuyo interés se justifica en base a los siguientes argumentos:

a).- los números naturales relativos son conceptos numéricos diferentes a los naturales y enteros. Las diferencias con los números naturales son obvias, mientras que con respecto a los números enteros se manifiestan: en el ámbito lógico-formal (inversión entre comparación y equivalencia en los procesos de construcción (González, 1995, pp. 214-219); cinco diferencias en las estructuras ordinales y algebraicas (op. cit., pp. 235-236)); en el ámbito fenomenológico (fenómenos diferentes (op. cit., pp. 260-277)) y en el ámbito cognitivo (los sujetos establecen, como veremos, una diferenciación clara en lo que se refiere a sus estructuras ordinales).

b).- los números naturales relativos abren nuevas perspectivas en la investigación didáctica. Proporcionan una nueva distribución del campo conceptual aditivo (Op. cit., pp. 224-226) que modifica y mejora las clasificaciones sobre problemas aditivos de enunciado verbal (Op. cit., pp. 259-283), facilitan la revisión de investigaciones anteriores aportando nuevas explicaciones a los resultados obtenidos (Coquin-Viennot, 1985; Vergnaud, 1982, pp. 48-49) y constituyen un instrumento para el análisis del área problemática desde nuevos enfoques.

\footnotetext{
${ }^{6}$ Operación aditiva compuesta de una comparación, por la que se determina el orden de los valores comparados, y de una sustracción entre números naturales (aquélla de las dos que es posible en N). Presenta, por tanto, dos partes diferenciadas: la sustracción natural y el sentido de la misma.
} 
c).- los números naturales relativos son didácticamente necesarios y útiles. Su consideración puede resolver interrogantes que surgen en la práctica educativa, favorecer un tratamiento didáctico adaptado a las características del desarrollo cognitivo "natural", facilitar el paso de los naturales a los enteros y de la Aritmética al Álgebra y evitar errores y dificultades de los alumnos (Bell, 1982).

\section{Método}

Participantes

Se ha utilizado una muestra intencional de 77 sujetos elegidos de acuerdo con los siguientes criterios: $1^{\circ}$ ) Sujetos con un nivel mínimo de conocimientos matemáticos necesarios para responder a los cuestionarios; $2^{\circ}$ ) Sujetos con un alto nivel de rendimiento escolar y formación general; $3^{\circ}$ ) Sujetos de diferente procedencia sociocultural, edad y tipo de actividad. La composición de la muestra es la siguiente: 54 estudiantes de los siguientes niveles: $2^{\circ}$ curso de Secundaria (13- 14 años) (4); Tercer curso de Secundaria (14- 15 años) (8); Formación Profesional (2); Primer, segundo y tercer curso de los estudios universitarios de Maestro de Primaria (40); 23 no estudiantes: Profesores universitarios (10), personal laboral de la Universidad (5) y personas que realizan actividades fuera de la Universidad (8); Sexo: varones (14), hembras (63); domicilio: rural (25), urbano (52); edades entre 14 y 47 años.

\section{Aproximaciones}

El análisis completo del problema se ha realizado desde tres aproximaciones distintas: formal, fenomenológica y cognitiva, y siguiendo dos líneas metodológicas: una teórica y otra empírica.

En la fase teórica se han utilizado técnicas de metaanálisis cualitativo (Fernandez Cano,1995; Ogawa y Malen, 1991), siguiendo un proceso que denominamos análisis didáctico (González, 1995, pp. 58-62; González, 1998), y se ha tomado como marco teórico de referencia la teoría intuitivo-constructiva de las formas conceptuales científicas (Stegmüller, 1979). Bajo tales supuestos se ha realizado un análisis pormenorizado del dominio de aplicación elemental de las estructuras aditivas y ordinales de los números naturales y los números ente- 
ros (González, 1998, pp.185-189) que ha revelado la existencia de tres tipos de relaciones (comparaciones, transformaciones y combinaciones), tres tipos de medidas (naturales, enteras y las que hemos denominado "medidas naturales relativas") y dos tipos de números (naturales y enteros) que, como hemos visto, no satisfacen las condiciones formales necesarias para ser dominios numéricos isomorfos al conjunto de medidas naturales relativas y actuar como soporte para la resolución directa de los problemas aritméticos correspondientes (González, 1998, pp.194-200).

El estudio teórico pone en evidencia, por tanto, la necesidad didáctica de considerar un tercer sistema numérico que proporcione la adecuada cobertura a la tercera estructura métrica identificada (medidas naturales relativas), lo que obliga a caracterizar y dotar de entidad al nuevo dominio y separarlo del de aplicación de la estructura entera. Para ello se define el concepto de número natural relativo y se realiza la construcción semiformalizada de la estructura de semigrupo aditivo, no conmutativo, sin elemento neutro, con doble valor nulo y parcialmente ordenada (op. cit., pp. 189-200).

El proceso descrito y sus resultados se pueden resumir en los siguientes puntos, que han constituido las hipótesis sometidas a crítica en el estudio teórico completo:

I.- En el dominio de aplicación concreta usual de la estructura aditiva y ordinal de los números naturales y los números enteros, existe un subdominio caracterizado por la intervención de un tipo de medidas discretas relacionadas con la comparación de medidas naturales y a las que llamaremos "medidas naturales relativas", entre las que se puede establecer una estructura de orden parcial y una ley de composición interna aditiva específica.

II.- Existe un conjunto de números a los que llamaremos números naturales relativos que, con la adición y el orden convenientes, es isomorfo al conjunto de medidas naturales relativas.

III.- El conjunto de los números naturales relativos con la adición y el orden definidos, presenta las cinco diferencias estructurales básicas siguientes con respecto al grupo aditivo y ordenado de los números enteros:

1) orden parcial con inversión en la región negativa, en vez de un orden total;

2) con primer elemento en cada una de las regiones, en vez de sin primer ele-mento;

3) desconexión entre las regiones positiva y negativa; 
4) doble valor nulo, en vez de un cero único;

5) anulación-compensación aditiva (adición natural relativa), en vez de la adición entera.

IV.- Los números naturales relativos abren una nueva via de extensión aditiva y ordinal de los números naturales a los números enteros, integrándose junto a ellos en un modelo teórico que relaciona entre sí todos los elementos del dominio, regulando las estructuras aritméticas aditivas correspondientes.

V.- El modelo mencionado permite establecer una clasificación lógico-semántica de los problemas y situaciones del dominio, que amplían y precisan otras ya realizadas sobre los problemas aditivos de enunciado verbal.

En la fase empírica se ha dirigido la atención hacia la primera de las diferencias establecidas en el apartado III, es decir: orden total para los números enteros, orden parcial para los números naturales relativos, aunque desglosada en los cuatro aspectos siguientes:

a).- atribución de significados, signos y adjetivos duales a las regiones;

b).- comparación-valoración global de regiones;

c).- comparación de medidas con valores numéricos negativos;

d).- comparación de medidas con valores numéricos de diferente signo o región

De cada uno de ellos se ha construido un cuestionario y se ha aplicado a una muestra intencional elegida de acuerdo con las características del problema. Con los datos se ha realizado un estudio descriptivo transversal (Bisquerra, 1989, pp. 217 y sgs.) con enfoque de presente y un análisis de correspondencias (Cornejo, 1988). Los resultados obtenidos están a favor de la siguiente hipótesis: individuos con estudios superiores al nivel correspondiente a 14 años de edad dan un tratamiento semántico diferenciado a los números naturales relativos y a los números enteros sobre la base de sus diferencias ordinales cuando se presentan en situaciones elementales de comparación de medidas discretas.

En el presente artículo se expone el estudio realizado mediante el primero de los cuatro cuestionarios, es decir, el estudio sobre atribución de significados, signos y adjetivos duales a las regiones. La elección de esta primera parte de la investigación, aunque obedece a limitaciones de espacio, no sustrae información esencial sobre el trabajo completo, puesto que 
los tres cuestionarios restantes tienen la misma estructura, orientación y formato que el primero, los mismos tipos de situaciones, los resultados son similares y en el sentido de la hipótesis y todos atienden a las características de los estudios sobre diferencial semántico. En consecuencia, remitimos al lector al informe original (González, 1998) para una información detallada sobre el estudio completo.

\section{Instrumentos}

Se pretende observar si los sujetos dan una valoración semántica global diferente, mediante pares de términos polarizados o frases cortas, a situaciones expresadas bajo la forma de enunciados genéricos sin especificación numérica y en contextos concretos y familiares, en los que se propone la comparación entre una cantidad/medida "positiva" y otra "negativa", correspondientes ambas a la misma magnitud y en dos casos distintos: i) cuando las dos cantidades/medidas son naturales relativas (que denominaremos "situaciones relativas") y ii) cuando las dos cantidades/medidas son enteras (que denominaremos "situaciones enteras"). Nuestra conjetura mantiene que si los sujetos responden de manera diferente a estos dos tipos de situa $\neg$ ciones, a las que se les suele aplicar indistintamente las propiedades y operaciones de los números enteros, se podría afirmar la existencia de diferencias cognitivas, reforzándose empíricamente la pertinencia y la utilidad didáctica del campo conceptual de los números naturales relativos.

Como quedará justificado en los párrafos que siguen, entenderemos que los sujetos responden de manera diferente a dichas situaciones cuando elijan términos objetivos fijos (mayor-menor, anterior-posterior, etc.) para las comparaciones entre medidas enteras, si bien se admite que elijan también términos subjetivos (mejor-peor, etc.), y términos subjetivos o, en general, que impliquen ausencia de valoración objetiva fija (depende, etc.), para las comparaciones entre medidas naturales relativas. El propósito del estudio es, por tanto, el de establecer un diferencial semántico entre las medidas enteras y las naturales relativas respecto a la rela $\neg$ ción de orden, aunque con una función de indagación en la estructura cognitiva y no en aspectos actitudinales, como ha sido lo habitual en los estudios de este tipo (Osgood, Suci y Tannerbaum, 1957; Summers, 1982).

Para la elaboración del instrumento se ha seguido el siguiente proceso: 
1).- Un estudio piloto, caracterizado por la elaboración, aplicación a una muestra intencional y análisis de resultados de un cuestionario inicial exploratorio;

2).- Elaboración del cuestionario final que aparece en el anexo 1. El estudio definitivo se realizó mediante la aplicación del cuestionario final a una nueva muestra intencional.

\section{Criterios para la elaboración del instrumento}

El orden total de los enteros se traduce en que son comparables / valorables cualesquiera dos medidas enteras $\mathrm{y}$, en particular, las medidas pertenecientes a regiones diferentes. Dicha comparación / valoración se suele expresar mediante pares de términos opuestos pertenecientes a las siguientes categorías:

- términos ordinales o comparativos, que establecen una relación directa entre los elementos: mayor-menor; superior-inferior; anterior-posterior; mejor-peor, etc.; de ellos, unos son objetivos (mayor-menor; superior-inferior; anterior-posterior) y otros, subjetivos (mejor-peor).

- términos clasificatorios, que establecen una relación indirecta: positivonegativo; más-menos; bueno-malo; etc., siempre que exista un convenio que conecte ambas categorías mediante otra intermedia que establece el orden; de ellos, unos son objetivos (másmenos; positivo-negativo; mayor que cero-menor que cero), y otros subjetivos (bueno-malo; fácil-difícil; alto-bajo).

Por el contrario, en la estructura de orden parcial de los números naturales relativos sólo son comparables las medidas del mismo signo (Ej.: una ganancia de 3 es mayor que una ganancia de 2, pero no es mayor que una pérdida de 2, que, en todo caso, será mejor). Aquí, la comparación / valoración objetiva de medidas de diferente signo es inadecuada, siendo admisibles, únicamente, las siguientes opciones: a) comparación / valoración fija aunque subjetiva (Ejemplos: "mejor / peor"; "fácil / difícil", etc.), b) comparación / valoración variable, dependiente de las circunstancias y del contexto (Ejemplo: "depende del caso"), c) independencia total en cualquier caso (Ejemplo: "son independientes"); las tres se pueden resumir en una sola: ausencia de valoración objetiva fija, en contraposición a lo que ocurre para la estructura de orden total de los números enteros . 


\section{Contenido y estructura del cuestionario}

El cuestionario se ha configurado en base a dos categorizaciones cruzadas:

a).- Columnas: tipo de valoración mediante expresión lingüística, con las siguientes categorías:

1).- valoración objetiva fija mediante pares de términos objetivos opuestos. Se han empleado para esta categoría los siguientes: "positivo (a) - negativo (a) (signo + / signo )"; "mayor que cero - menor que cero"; "superior - inferior" y "anterior - posterior";

2).- ausencia de valoración objetiva fija, que incluye el resto de valoraciones: va $\neg$ lo-ración fija subjetiva, con tres pares de términos opuestos (“bueno (a) - malo (a) (positivo / negativo)"; "fácil - difícil"; "alto (a) - bajo (a)"), valoración variable o dependiente y ausencia de valoración fija ("depende del caso" y "son independientes y la valoración es indiferente en cualquier caso").

b).- Filas: tipo de situación que se somete a los encuestados para adjetivar o calificar, mediante los pares de términos o frases cortas de la categoría anterior, las dos regiones duales en que se divide el universo de valores. Es una variable cualitativa que presenta dos modalidades: situaciones de medidas enteras contrapuestas (situaciones enteras) y situaciones de medidas naturales relativas contrapuestas (situaciones relativas ), todas con la misma estructura: pareja de cantidades genéricas opuestas enunciadas verbalmente y separadas por una barra (ejemplo: "un índice que indica una bajada de la bolsa / uno que indica una subida").

En el cuestionario se incluyen en total 24 situaciones (11 enteras y 13 relativas). Las que ocupan las posiciones $2,3,5,8,10,11,13,16,17,18,19,21$ y 24 fueron seleccionadas como casos de situaciones re $\neg$ lativas. Las situaciones que ocupan las posiciones 1, 4, 6, 7, 9, 12, 14, 15, 20, 22 y 23 fueron seleccionadas como casos de medidas enteras. Hay que señalar que esta distinción no es siempre sencilla, ya que hay casos en los que se pueden combinar las dos caracterizaciones.

\section{Respuestas}


La conexión entre filas y columnas se realiza mediante una frase única que comienza en una situación - fila para terminar, por medio de una palabra o frase corta de enlace $(\mathrm{Ej}$.: "son situaciones contrarias . . que normalmente se valoran mediante el par de términos:") en un hiato gramatical en el que situar uno o varios valores de los contemplados en las columnas. La composición: par de medidas opuestas (fila) - frase de enlace - par de palabras o frase corta (columna) da lugar a un texto que puede o no tener sentido. Al encuestado se le pide que marque, para cada fila, todas las modalidades columna que dan lugar a frases con sentido. La variable dependiente es el tipo de conexión, con dos modalidades: elegida o no elegida, codificadas mediante 1 y 0 respectivamente.

\section{Procedimiento}

El cuestionario se aplicó a cinco grupos de estudiantes, dos de ellos completos $\left(8^{\circ} \mathrm{de}\right.$ E.G.B. y $1^{\mathrm{o}}$ de B.U.P.), en horas de clase y sin ningún tipo de aclaración. A profesores y no estudiantes se les entregó individualmente, solicitando su cumplimentación en el plazo más breve posible. A todos se les hizo la petición de que trataran de responder a todas las cuestiones.

Se recogieron 123 cuestionarios cumplimentados, de los que se hizo una selección eliminando quince de ellos con cinco o más respuestas en blanco o que habían sido respondidos de forma sistemática. Del resto, hasta un total de 108, se hizo un nuevo muestreo intencional mediante una segunda aplicación, más restrictiva, de los criterios utilizados para la elección de la muestra inicial; de esta nueva selección resultaron los 77 que iban a ser utilizados en el estudio.

Los resultados se han recogido en tablas de frecuencias y diagramas. Para el análisis de datos se ha utilizado la hoja de cálculo Excel 2.2, el paquete SPSS y el programa SAS en el ordenador VAX/VMS versión v5.5-2 del Centro de Proceso de datos de la Universidad de Málaga.

\section{Análisis estadísticos}

\section{Análisis descriptivo}


En la tabla 1 se reflejan las frecuencias absolutas de las respuestas al cuestionario, figurando en sombreado las partes que, de acuerdo con las predicciones teóricas enunciadas, deberían recoger en total el mayor número de respuestas por fila en cada una de las 24 situaciones: en el bloque I las situaciones enteras (respuestas objetivas o de tipo 1) y en el bloque II las naturales relativas (respuestas sub $\neg$ jetivas o de tipo 2) y otras respuestas (ausencia de valoración, depende del caso, de tipo 3).

Tabla 1. Frecuencias absolutas y zonas de respuesta esperada.

\begin{tabular}{|c|c|c|c|c|c|c|c|c|c|c|c|c|}
\hline & \multicolumn{5}{|c|}{ bloque I } & \multicolumn{6}{|c|}{ bloque II } & \\
\hline & \multicolumn{4}{|c|}{ res pues tas ti po 1} & \multirow[b]{2}{*}{ SUMA } & \multicolumn{3}{|c|}{ res pues tas tipo 2} & \multicolumn{2}{|c|}{ res pues tas ti po 3} & & \\
\hline & $\begin{array}{l}\text { positivo } \\
\text { negativ o }\end{array}$ & $\begin{array}{l}\text { mayor } \\
\text { menor }\end{array}$ & $\begin{array}{l}\text { sup erio } 1 \\
\text { inferior }\end{array}$ & $\begin{array}{c}\text { ant erior } \\
\text { po sterio n }\end{array}$ & & $\begin{array}{c}\text { bueno } \\
\text { malo }\end{array}$ & $\begin{array}{c}\text { fácil } \\
\text { difícil }\end{array}$ & $\begin{array}{l}\text { alto } \\
\text { bajo }\end{array}$ & dep end & ind epend & SUMA & $\begin{array}{l}\text { SUMA } \\
\text { TOTAL }\end{array}$ \\
\hline $\mathrm{x}_{1}$ & 68 & B2 & Iflem & on & Itifin: & 53 & 8 & 12 & 1 & 0 & 74 & 185 \\
\hline $\mathrm{x}_{2}$ & 23 & 11 & 4 & 1 & 39 & 48 & In & on & Ille & tilin & 8 & 119 \\
\hline $\mathrm{x}_{3}$ & 47 & 8 & 9 & 0 & 64 & 58 & (m) & 6 & 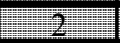 & (1) & 8 & 146 \\
\hline $\mathrm{x}_{4}$ & (n) & illin & 4 & 68 & 7 & 3 & 4 & 0 & 11 & 0 & 18 & 93 \\
\hline $\mathrm{x}_{5}$ & 44 & 7 & 18 & 2 & 71 & Bis & 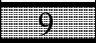 & 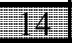 & Pen & Bi & 68 & 139 \\
\hline $\mathrm{x}_{6}$ & 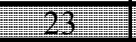 & (6) & 26 & then & tittis & 16 & 1 & 32 & 10 & 0 & 59 & 172 \\
\hline $\mathrm{x}_{7}$ & 霓 & 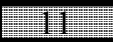 & 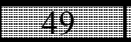 & 籍 & (173) & 7 & 0 & 56 & 6 & 0 & 69 & 142 \\
\hline $\mathrm{x}_{8}$ & 3 & 0 & 26 & 6 & 35 & 83 & Bs & 385 & 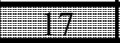 & 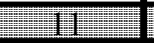 & 69 & 104 \\
\hline $\mathrm{x}_{9}$ & 4 & (9) & (1) & 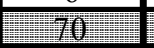 & 88 & 5 & 1 & 1 & 3 & 1 & 11 & 99 \\
\hline $\mathrm{x}_{10}$ & 7 & 1 & 16 & 3 & 27 & 12 & (B) & 48 & 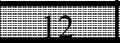 & Bis & 88 & 115 \\
\hline $\mathrm{x}_{11}$ & 14 & 2 & 14 & 1 & 31 & 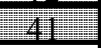 & 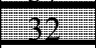 & 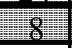 & 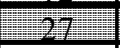 & 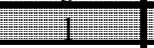 & 100 & 140 \\
\hline $\mathrm{x}_{12}$ & 58 & 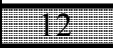 & (I) & Bis & 80 & 54 & 17 & 5 & 6 & 0 & 82 & 162 \\
\hline $\mathrm{x}_{13}$ & 12 & 6 & 14 & 7 & 39 & III & 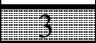 & 倍 & 29) & 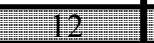 & 64 & 103 \\
\hline $\mathrm{x}_{14}$ & 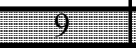 & 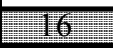 & (32) & 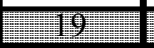 & 96 & 7 & 0 & 35 & 0 & 5 & 47 & 143 \\
\hline $\mathrm{x}_{15}$ & 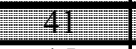 & n10 & 25 & 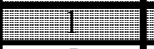 & 77 & 36 & 25 & 16 & 3 & 1 & 81 & 158 \\
\hline $\mathrm{x}_{16}$ & 15 & 8 & 11 & 5 & 39 & Billit & 6) & 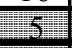 & 20) & IIIIII & 6ititi: & 106 \\
\hline $\mathrm{x}_{17}$ & 21 & 32 & 24 & 3 & 80 & 8 & Bul & $\frac{\pi}{186}$ & 119 & 2 & T) & 159 \\
\hline $\mathrm{x}_{18}$ & 15 & 2 & 5 & 5 & 27 & 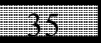 & D2 & 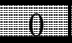 & IIIII: & 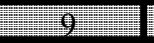 & 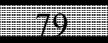 & 106 \\
\hline $\mathrm{x}_{19}$ & 18 & 2 & 19 & 7 & 46 & 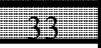 & $\varphi$ & Bs" & 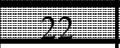 & 6 & (n) & 121 \\
\hline $\mathrm{x}_{20}$ & His" & (S) & 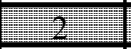 & 60 & 80 & 15 & 2 & 0 & 6 & 2 & 25 & 105 \\
\hline $\mathrm{x}_{21}$ & 11 & 4 & 26 & 1 & 42 & 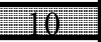 & 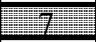 & (1) & 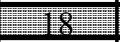 & 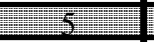 & 8 & 129 \\
\hline $\mathrm{x}_{22}$ & stitin & (9) & 24 & Bu & (1) & 44 & 16 & 25 & 4 & 0 & 89 & 182 \\
\hline$x_{23}$ & 20 & 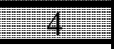 & 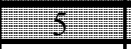 & 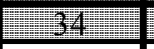 & 6 & 22 & 7 & 2 & 20 & 4 & 55 & 118 \\
\hline$x_{24}$ & 32 & 4 & 21 & 10 & 67 & S5) & 䤃 & 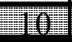 & Bal & 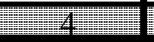 & 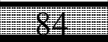 & 151 \\
\hline
\end{tabular}

En los gráficos de la figura 2 se representan, mediante diagramas de barras dobles (en negro y sombreada para los dos bloques de respuesta), las frecuencias absolutas de las respuestas objetivas y subjetivas a las situaciones naturales relativas y a las situaciones enteras, lo cual pemite apreciar gráficamente el predominio de los tipos de respuestas para cada situación. 
Cu estionario a.1.- Frecu enci as abs olut as de respu estas de ti po objetivo y subjetivo a situaciones enteras.

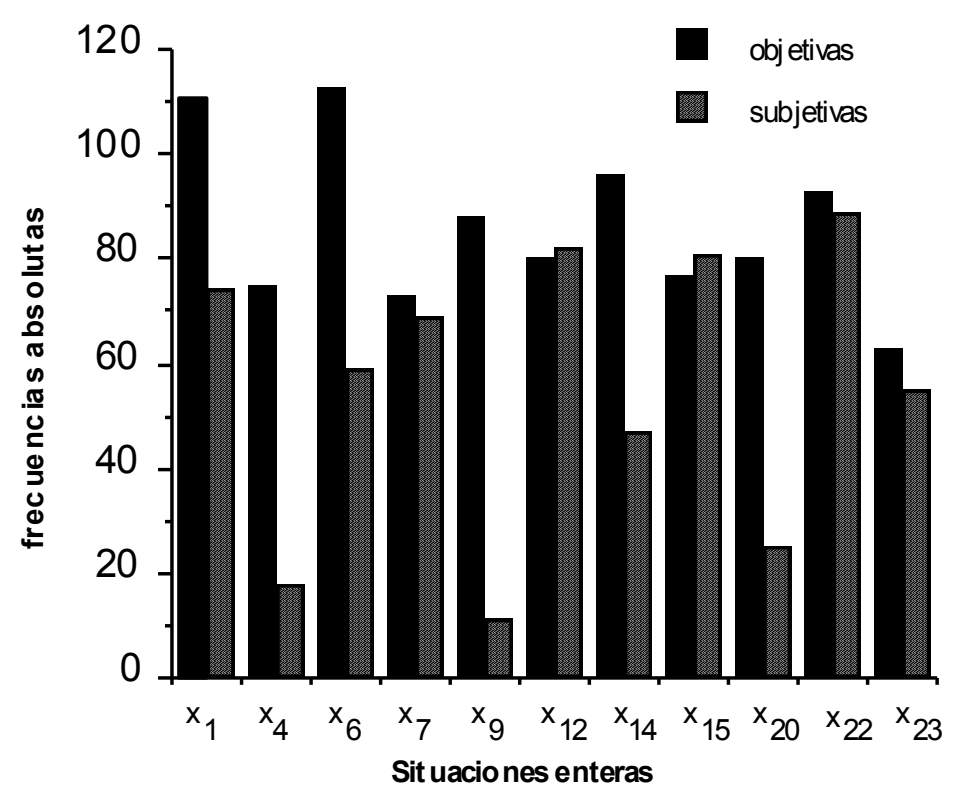

Cuestionario a.1.- Frecu enci as absolut as de respu estas de tipo objetivo y subjetivo a situaciones relativas.

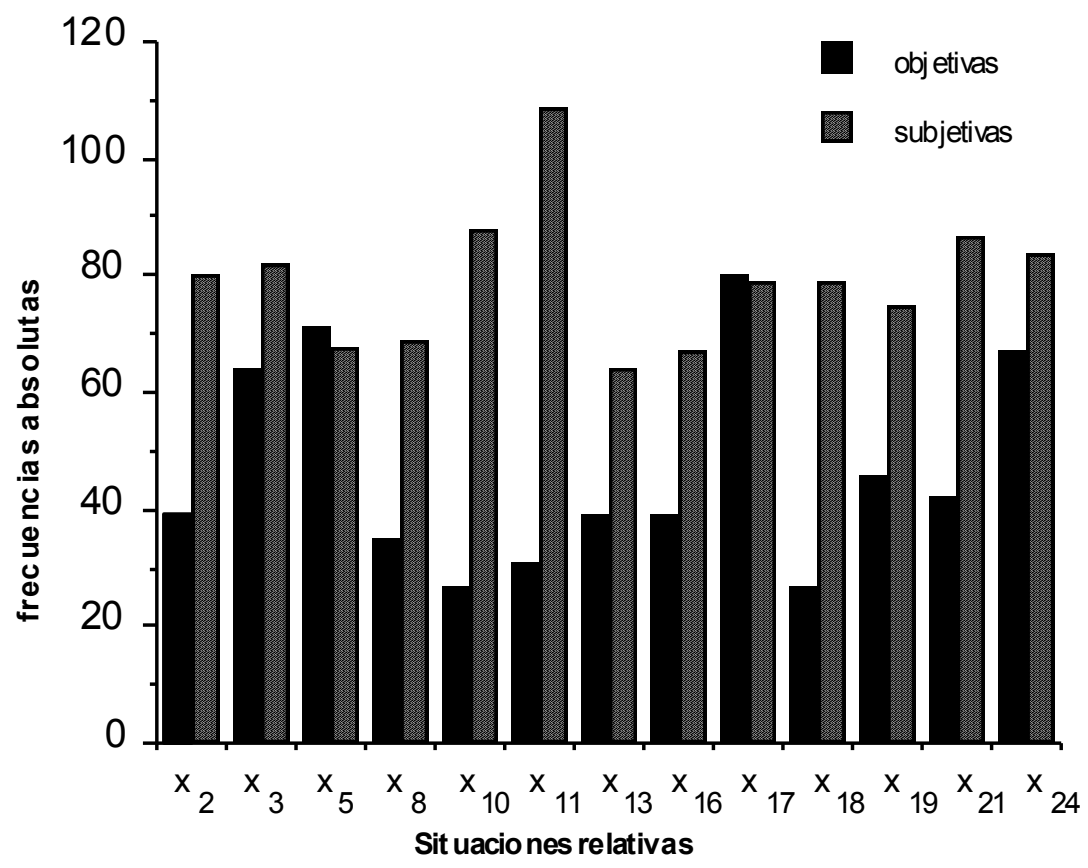

Figura 2. Frecuencias absolutas de respuestas a los dos tipos de situaciones

Como se deduce de una inspección de la tabla 1, y se puede apreciar en la figura 2, la tendencia en 20 de las 24 situaciones es de agrupamiento de las situaciones enteras en torno a la valoración objetiva y de las situaciones naturales relativas en torno a la ausencia de valoración objetiva fija, lo que representa el $83,33 \%$ del total. En las situaciones enteras x12 y x15 
se presenta un predominio de las respuestas de tipo 2 (subjetivas), lo cual resulta admisible en nuestra conjetura, mientras que en las dos situaciones restantes, x5 y x17, la tendencia de agrupamiento es inversa de la prevista, si bien con diferencias pequeñas; estas situaciones corresponden al $8,33 \%$ del total.

Esta primera descripción genérica parece apuntar en la dirección de sostener nuestra conjetura. Sin embargo, las diferencias entre la valoración objetiva fija y la ausencia de valoración objetiva fija no son siempre tan acusadas como sería de desear; se producen algunos resultados que necesitan de interpretación, por lo que pasamos a una descripción más pormenorizada.

\section{Análisis comparativo puntual}

Se han calculado los tamaños del efecto por cada situación, comparando la valoración objetiva (bloque I) frente a la valoración subjetiva o ausencia de valoración (bloque II). En la tabla 2 se puede observar que las concordancias más altas son las esperadas según la prediccción de la hipótesis: 15 de ellas no son debidas al azar, con un error tipo $\square<0.01$, y cinco son débiles (las correspondientes a los items: x3, x7, x22, x23 y x24). Las discordancias se presentan sólo en cuatro items (x5, x12, x15 y x17), con tamaños del efecto nimios y nula significación estadística.

\section{Análisis comparativo global}

Se han calculado la media aritmética y la desviación típica a las cua $\neg$ tro variables que resultan de combinar los dos tipos de respuesta (valoración objetiva y valoración subjetiva o ausencia de valoración) con los dos tipos de situaciones. En la figura 3 se han representado los intervalos para las cuatro variables, observándose que son apreciables las diferencias entre las respuestas objetivas y subjetivas así como entre las respuestas objetivas a los dos tipos de situaciones. 
Tabla 2. Tamaño del efecto y contraste inferencial

\begin{tabular}{|c|c|c|c|c|}
\hline Items & (tamaño del efecto) & Concordancia & $\chi^{2}$ & $\mathbf{P}$ \\
\hline 1 & 0.41 & si & 7.40 & $0.01^{*}$ \\
\hline 2 & -0.73 & si & 14.13 & $0.00^{*}$ \\
\hline 3 & -0.25 & si & 2.22 & 0.14 \\
\hline 4 & 1.55 & si & 39.94 & $0.00^{*}$ \\
\hline 5 & 0.04 & no & 0.06 & 0.81 \\
\hline 6 & 0.66 & si & 16.95 & $0.00^{*}$ \\
\hline 7 & 0.06 & si & 0.11 & 0.74 \\
\hline 8 & -0.69 & si & 11.12 & $0.00^{*}$ \\
\hline 9 & 2.47 & si & 59.89 & $0.00^{*}$ \\
\hline 10 & -1.25 & si & 32.36 & $0.00^{*}$ \\
\hline 11 & -1.34 & si & 43.46 & $0.00^{*}$ \\
\hline 12 & -0.02 & no & 0.02 & 0.89 \\
\hline 13 & -0.50 & si & 6.07 & $0.01^{*}$ \\
\hline 14 & 0.73 & si & 16.79 & $0.00^{*}$ \\
\hline 15 & -0.05 & no & 0.10 & 0.75 \\
\hline 16 & -0.55 & si & 7.40 & $0.01^{*}$ \\
\hline 17 & 0.01 & no & 0.01 & 0.92 \\
\hline 18 & -1.13 & si & 25.51 & $0.00^{*}$ \\
\hline 19 & -0.49 & si & 6.95 & $0.01^{*}$ \\
\hline 20 & 1.23 & si & 28.81 & $0.00^{*}$ \\
\hline 21 & -0.74 & si & 15.70 & $0.00^{*}$ \\
\hline 22 & 0.04 & si & 0.09 & 0.76 \\
\hline 23 & 0.14 & si & 0.54 & 0.41 \\
\hline 24 & -0.23 & si & 1.91 & 0.17 \\
\hline
\end{tabular}

En lo que respecta a la variabilidad es de destacar la homogeneidad de las respuestas subjetivas o ausencia de valoración a las situaciones relativas, que contrasta con la dispersión de los valores de este mismo tipo de respuesta en el caso de las situaciones enteras. Esta dispersión se puede explicar por la coexistencia en el cuestionario de situaciones enteras clásicas (temperaturas, cronología, índices de bolsa, etc.) con situaciones enteras poco conocidas (golf o gastos e ingresos familiares). Hemos de recordar que dentro de nuestra hipótesis no está excluido el tratamiento de situaciones enteras mediante términos subjetivos, pero sí estan ex- 
cluidos tanto la ausencia de valoración para situaciones enteras como el tratamiento de situaciones relativas mediante términos objetivos, lo que queda patente en el gráfico de la figura 3 .

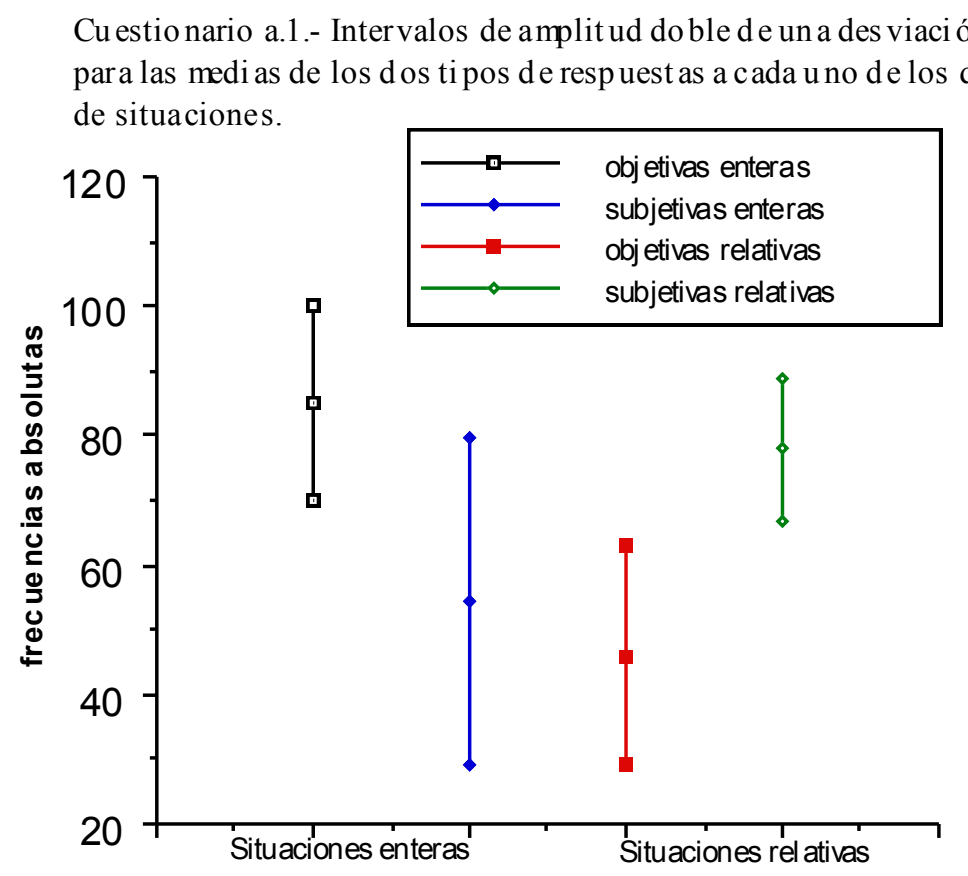

Figura 3.Intervalos para los tipos de cuestiones.

Análisis de correspondencias

Con los datos originales, se ha construído una tabla de contingencia for $\neg$ mada por las 24 situaciones dispuestas por filas y por tres grupos de respuestas en las columnas (grupo 1: valoraciones objetivas; grupo 2: valoraciones subjetivas; grupo 3: ausencia de valoración fija). Con estos datos se ha realizado un análisis de correspon $\neg$ dencias en el que aparecen dos factores que explican el 100\% de la inercia, con el $59.5 \%$ y el $40.5 \%$, respectivamente.

El análisis de las frecuencias observadas queda resumido en la tabla 3, en la que se observa que la calidad de todos los items es 1, lo que indica que están bien representados en ese espacio bidimensional. Las frecuencias de respuestas oscilan en el intervalo [0.029, 0.058], lo que indica una gran uniformidad. La mayor contribución a la inercia total corresponde a: x4, x9, x10, x11, x13, x16, y x20, mientras que: x5, x7, x12, x17 y x24 tienen una contribución nula o ínfima. 
Tabla 3. Estadísticos básicos para el análisis de correspondencias

\begin{tabular}{cccccc}
\hline Item & $\begin{array}{c}\text { Frecuencia sobre } \\
\text { tamaño de la muestra }\end{array}$ & $\begin{array}{c}\text { Calidad de } \\
\text { inclusión en e } \\
\text { plano }\end{array}$ & $\begin{array}{c}\text { Contribu- } \\
\text { ción de la } \\
\text { fila a la } \\
\text { inercia total }\end{array}$ & $\begin{array}{c}\text { Correlación } \\
\text { del item con } \\
\text { el eje } 1\end{array}$ & $\begin{array}{c}\text { Correlación } \\
\text { del item con } \\
\text { el eje } 2\end{array}$ \\
\hline $\mathrm{x}_{1}$ & 0.058 & 1.00 & 0.007 & 0.900 & 0.100 \\
$\mathrm{x}_{2}$ & 0.037 & 1.00 & 0.004 & 0.356 & 0.644 \\
$\mathrm{x}_{3}$ & 0.046 & 1.00 & 0.007 & 0.089 & 0.911 \\
$\mathrm{x}_{4}$ & 0.029 & 1.00 & 0.014 & 0.344 & 0.656 \\
$\mathrm{x}_{5}$ & 0.043 & 1.00 & 0.000 & 0.857 & 0.143 \\
$\mathrm{x}_{6}$ & 0.054 & 1.00 & 0.006 & 0.848 & 0.152 \\
$\mathrm{x}_{7}$ & 0.044 & 1.00 & 0.002 & 0.526 & 0.474 \\
$\mathrm{x}_{8}$ & 0.033 & 1.00 & 0.009 & 0.852 & 0.148 \\
$\mathrm{x}_{9}$ & 0.031 & 1.00 & 0.020 & 0.654 & 0.346 \\
$\mathrm{x}_{10}$ & 0.036 & 1.00 & 0.010 & 0.490 & 0.510 \\
$\mathrm{x}_{11}$ & 0.044 & 1.00 & 0.013 & 0.873 & 0.127 \\
$\mathrm{x}_{12}$ & 0.051 & 1.00 & 0.003 & 0.336 & 0.664 \\
$\mathrm{x}_{13}$ & 0.032 & 1.00 & 0.027 & 0.513 & 0.487 \\
$\mathrm{x}_{14}$ & 0.045 & 1.00 & 0.007 & 0.944 & 0.056 \\
$\mathrm{x}_{15}$ & 0.049 & 1.00 & 0.004 & 0.286 & 0.714 \\
$\mathrm{x}_{16}$ & 0.033 & 1.00 & 0.016 & 0.622 & 0.378 \\
$\mathrm{x}_{17}$ & 0.050 & 1.00 & 0.000 & 0.032 & 0.968 \\
$\mathrm{x}_{18}$ & 0.033 & 1.00 & 0.007 & 0.872 & 0.128 \\
$\mathrm{x}_{19}$ & 0.038 & 1.00 & 0.006 & 0.823 & 0.177 \\
$\mathrm{x}_{20}$ & 0.033 & 1.00 & 0.010 & 0.575 & 0.425 \\
$\mathrm{x}_{21}$ & 0.040 & 1.00 & 0.005 & 0.945 & 0.055 \\
$\mathrm{x}_{22}$ & 0.057 & 1.00 & 0.005 & 0.444 & 0.556 \\
$\mathrm{x}_{23}$ & 0.037 & 1.00 & 0.005 & 0.084 & 0.916 \\
$\mathrm{x}_{24}$ & 0.047 & 1.00 & 0.003 & 0.052 & 0.948 \\
\hline & & & & & \\
\hline
\end{tabular}

En la tabla 4 se indican los estadísticos correspondientes a los tipos de valoración, mientras que en la figura del Anexo 2 se representa la distribución de las proyecciones de los puntos fila y de los puntos columna sobre el plano de coordenadas. 
Tabla 4. Estadísticos relativos a los tipos de valoración consideradas

\begin{tabular}{cccccccc}
\hline $\begin{array}{c}\text { Tipo de } \\
\text { valoración }\end{array}$ & $\begin{array}{c}\text { Frecuencia } \\
\text { sobre el ta- } \\
\text { maño de la } \\
\text { muestra }\end{array}$ & $\begin{array}{c}\text { Coordenada } \\
\text { respecto al } \\
\text { eje 1 }\end{array}$ & $\begin{array}{c}\text { Correlación } \\
\text { respecto al } \\
\text { factor I }\end{array}$ & $\begin{array}{c}\text { Inercia rela- } \\
\text { tiva de la } \\
\text { fila al eje 1 }\end{array}$ & $\begin{array}{c}\text { Coordenada } \\
\text { respecto al } \\
\text { eje 2 }\end{array}$ & $\begin{array}{c}\text { Correlación } \\
\text { respecto al } \\
\text { factor 2 }\end{array}$ & $\begin{array}{c}\text { Inercia } \\
\text { relativa de } \\
\text { la fila al eje } \\
2\end{array}$ \\
\hline objetiva & 0.487 & -0.293 & 0.789 & 0.368 & -0.152 & 0.211 & 0.145 \\
subjetiva & 0.402 & 0.146 & 0.176 & 0.076 & 0.317 & 0.824 & 0.522 \\
ausencia & 0.111 & 0.753 & 0.711 & 0.556 & -0.480 & 0.289 & 0.333 \\
\hline
\end{tabular}

Del analisis de correspondencias realizado obtenemos las siguientes conclusiones:

a).- La dimensión 1 es el resultado de una mayor contribución de los puntos columna 1 y 3, mientras que la dimensión 2 tiene una mayor contribución del punto columna 2, que se refiere a las valoraciones de tipo subjetivo. Los términos de valoración objetiva contribuyen a la inercia de la dimensión 1, los de valoración subjetiva a la de la dimensión 2 y los que indican ausencia de valoración contribuyen a la inercia en am $\neg$ bas dimensiones, con ventaja a favor de la dimensión 1.

b).- Existe oposición entre el punto 1 y los puntos 2 y 3, separados por el eje de la dimensión 2; oposición que se produce entre valoraciones objetivas fijas y otros tipos de valoraciones englobados bajo la categoría "ausencia de valoración fija".

c).- El eje vertical se sitúa como línea de separación entre las situaciones con valo $\neg$ ración objetiva y las situaciones con ausencia de valoración fija, con excepción de los enunciados x3, x5, x23 y x24. Las primeras toman valores negativos para las abcisas, mientras que las segundas se sitúan en puntos con abcisas positivas, a la derecha del mencionado eje.

d).- Considerando sólo la primera dimensión se constatan las siguientes asociaciones:

En torno a las valoraciones de tipo 1:

- Las situaciones x1, x4, x6, x9, x14 y x20, todas ellas enteras, presentan valoracio $\neg$ nes marcadamente objetivas (grupo 1).

- Las situaciones x5, x7, x17, x22 y x23, de las cuales x5 y x17 son relativas, se en $\neg$ cuentran situadas en una zona intermedia entre el punto $1 \mathrm{y}$ el punto 2 , presentando valoraciones equilibradas entre las del grupo 1 (valoraciones objetivas) y las del grupo 2 (valoraciones subjetivas fijas), con una ligera ventaja a favor de las del tipo 1. 
En torno a las valoraciones de tipo 2:

- Las situaciones x3, x12, x15 y x24, de las que x12 y x15 son enteras, se encuentran situadas en una zona intermedia entre el punto 1 y el punto 2 , presentando valoraciones equilibradas entre las del grupo 1 y las del grupo 2, con una ligera ventaja a favor de las del tipo 2.

- Las situaciones x2, x10, x11, x18 y x21, todas ellas relativas, se encuentran clara $\neg$ mente asociadas al punto 2 correspondiente a las valoraciones de tipo subjetivo fijo.

En torno a las valoraciones de tipo 3:

- Las situaciones relativas x8 y x19 se encuentran en una zona intermedia entre los puntos 2 y 3 , por lo que su valoración se encuentra entre la subjetiva fija y la ausencia de valoración fija.

- Las situaciones relativas x13 y x16 son las que presentan mayor tendencia hacia la valoración dependiente o la ausencia de una valoración concreta.

e).- Son cuatro las situaciones, unas enteras y otras relativas, que no están bien repre $\neg$ sentadas en el factor o dimensión 1, dada su baja correlación $(|\mathrm{r}|<0.25)$, pero que, en cambio, si están muy bien representadas en el eje 2; son las situaciones x3, x17, x23 y x24, que deberán ser reconsideradas al ser las que reducen las posibilidades confirma $\neg$ torias en el modelo propuesto.

\section{Resultados}

De los análisis realizados y del exámen de los datos destacamos las siguientes consideraciones:

A).- En el conjunto de las 11 situaciones de comparación de medidas enteras encontramos que:

- se produce una valoración objetiva superior a la subjetiva en el $81,8 \%$ de las situaciones, a las que los sujetos responden mediante términos propios de una relación de orden total;

- las frecuencias de las respuestas de tipo 3 (ausencia de valoración) son muy ba $\neg$ jas en todos los casos, excepto en la situación x23; las frecuencias de las valoraciones objetivas son superiores a las de los otros dos tipos, excepto en dos casos: x12 y x15, en los que no hay 
concordancia con lo previsto; en el resto de los casos si hay concordancia y en 6 de ellos no es debida al azar;

- las correlaciones de las 11 situaciones enteras con respecto al eje 1 son superio $\neg$ res a 0.30, excepto la situación x 23 (adelanto-atraso en una cita), que es también la excepción a la separación establecida por el eje vertical entre las situaciones con valo $\neg$ ración objetiva fija y otras situaciones.

- hay tres tipos de situaciones enteras en función del nivel alcanzado en la valoración objetiva:

* situaciones con valoración fuertemente objetiva: saldo en cuenta bancaria (x1); tiempo respecto a un momento o fecha (x4, x9 y x20); temperaturas (x6) y posición de los pisos respecto a la planta baja (x14). Todas tie $\neg$ nen una correlación alta con el eje 1 , excepto la $\mathrm{x} 4$.

* situaciones con valoración objetiva superior a la subjetiva: nivel del mar (x7) e índice de bolsa (x22); la correlación de ambas con el eje 1 es aceptable.

* situaciones con valoración subjetiva ligeramente superior a la objetiva: balance economía familiar (x12) y juego del golf (x15); la correlación de ambas con el eje 1 es baja.

- las discrepancias con respecto a lo esperado son aceptables, de manera que, salvo el caso de la situación x23, la hipótesis se verifica para la comparación de medidas enteras. En efecto:

En general, los términos subjetivos son de uso extendido y, con frecuencia, más comunes y familiares que los términos objetivos, lo que puede suponer que las diferencias entre los dos tipos de respuesta sea menor en el caso de las situaciones enteras que en el de las relativas, e incluso que en algunas situaciones enteras las respuestas subjeti $\neg$ vas lleguen a superar a las objetivas.

Aunque las diferencias son muy pequeñas, las situaciones enteras x12 y x15, que representan el $18,2 \%$, se valoran preferentemente y de forma global mediante términos no marcadamente objetivos. Esta falta de consistencia en la concordancia puede ser debida a que la situación x12 (balances económicos familiares) emplea un término ("balance”) de uso poco frecuente e implica variables de los dos tipos: balance (variable entera) y gastos e ingresos (variables naturales relativas). Por otra parte, creemos que la situación x15 (golf) se presta a 
confusión por el desconocimiento del juego y la peculiaridad del sistema de puntuaciones, a veces contradictorias con lo usual. En cualquier caso, parece razonable que en estas dos situaciones, ante la duda, los sujetos prefieran utilizar términos válidos pero menos comprometidos y rigurosos que los que han sido clasificados como objetivos. Por último, la situación x23 ("minutos que han pasado/ minutos que faltan") tiene un planteamiento confuso; emplea verbos contrapuestos que se pueden considerar desde un punto de vista relativo, lo que puede haber propiciado la alta frecuencia de valoraciones de tipo 3.

B).- En las 13 situaciones de comparación de medidas naturales relati $\neg$ vas encontramos que:

- se produce una ausencia de valoración objetiva superior a la valoración objetiva en el $84,6 \%$ de las situaciones; predominan las respuestas que expresan una valoración subjetiva o una ausencia de valoración. En el 15,4 \% restante hay equilibrio entre las valoraciones objetivas y las que no lo son.

- las valoraciones de tipo 3 (ausencia de valoración) tienen frecuencias superiores al $10 \%$ del total, excepto en las situaciones $\mathrm{x} 3 \mathrm{y}$ x 24 ; las valoraciones objetivas son inferiores a la suma de las otras dos, excepto en los casos x 5 y x17, en los que se da la igualdad y no se produce concordancia; en el resto de los casos sí hay concordancia y en 9 de ellos no es debida al azar.

- las correlaciones de las 13 situaciones con respecto al eje 1 son superiores a 0.30 , excepto las situaciones $\mathrm{x} 3, \mathrm{x} 17 \mathrm{y}$ x 24 . La primera y la tercera tienen un porcentaje bajo de valoraciones de tipo 3 y la segunda tiene un por $\neg$ centaje de valoraciones objetivas superior al resto.

- encontramos cuatro tipos de situaciones relativas en función de los distintos tipos de respuestas:

*valoración fuertemente subjetiva: faltar / sobrar, subir / bajar (escalones y metros en globo), aumentar / disminuir y encontrar / perder (x2, x10, x11, x18 y x21). Todas tienen una correlación aceptable con el eje 1.

*valoración subjetiva superior a la objetiva con ausencia de valoración baja: ganancia / pérdida (x3) y descuento / recargo sobre un precio (x24); la correlación con el factor I es muy baja y, aunque presentan concordancia, su error es mayor que 0.01 . 
*predominio de la ausencia de valoración objetiva fija (valoración subjetiva similar a la objetiva y a la ausencia de valoración) (las frecuencias de los tres tipos de respuestas es prácticamente la misma): subida / bajada, salida / entrada y ascenso / descenso (x8, x13, x16 y x19); las correlaciones con el eje 1 son altas y la concordancia no es debida al azar en ningún caso.

*ausencia de valoración objetiva igual a la valoración objetiva (las frecuencias acumuladas de la valoración subjetiva y la ausencia de valoración igualan a las frecuencias de la valoración objetiva): situaciones x5 y x17 (ingresos-reintegros y variación de temperaturas), que suelen presentarse como enteras y es menos usual plantearlas como relativas. Son los dos únicos casos en los que no hay concordancia y la correlación con el eje 1 es inapreciable.

- Podemos asegurar que se verifica la hipótesis de partida salvo en el caso de las situaciones $\mathrm{x} 5$ y x17, aunque estas discrepancias con respecto a lo esperado admiten interpretación. En efecto:

La situación x 5 se refiere a la valoración de ingresos-reintegros en una cuenta bancaria, cuya respuesta más frecuente ha sido "positivo (signo +), negativo (signo -)". Entendemos que esto es debido a que los ingresos y reintegros bancarios se pueden considerar de dos maneras diferentes e igualmente válidas: como acciones opuestas que se conocen vulgarmente como "meter-sacar" dinero (estructura natural relativa), sentido con el que hemos incluído esta situación en el cuestionario, y como un apunte que hace el banco mediante un número con signo y que indica una cantidad a añadir o a restar a la ya existente (interpretación aso $\neg$ ciada al saldo bancario y, por tanto, enmarcada en una estructura entera). Esta es la interpretación que ha predominado en este caso.

El resultado de la situación x17 creemos que se explica por la utilización prioritaria de la escala de temperaturas. Una variación de temperatura, además de ser de uso poco frecuente, se encuentra indeterminada hasta que sea asociada con la temperatura a partir de la que se ha producido la variación. De esta manera es frecuente asociar un aumento de temperatura con una temperatura "mayor" o "superior"; de aquí es fácil pasar a "mayor que cero" o "positivo $($ signo + )" sustituyendo la estructura de orden parcial por la de orden total correspondiente, que en este caso es más familiar. 


\section{Discusión}

Los resultados del cuestionario analizado, al igual que los del resto del estudio empírico (González, 1995, pp. 341-390), aportan indicios a favor de las hipótesis enunciadas y apuntan en la dirección de la bondad de los planteamientos teóricos, es decir, las diferencias entre los campos conceptuales comparados no son sólo lógico-formales y fenomenológicas, sino que también se manifiestan desde el punto de vista cognitivo.

Para nosotros es evidente que la investigación carecería de interés si existiera homogeneidad, desde el punto de vista cognitivo, entre los diferentes fenómenos, situaciones y problemas del dominio, es decir, si los sujetos consideraran la estructura numérica entera como la única idónea para ser aplicada de manera "natural", sin dificultades y por igual a todas las situaciones apropiadas del campo aditivo. Pero esto no es así, el estudio pone en evidencia la heterogeneidad de significados, términos y estructuras asociadas a los dos tipos de situaciones y fenómenos considerados: los que se modelizan mediante la estructura entera y los que lo hacen mediante la estructura natural relativa; diferenciación que se materializa en la utilización preferente de términos objetivos y estrechamente ligados a una estructura de orden total (positivo-negativo, mayor que cero-menor que cero, etc.) cuando se hacen comparaciones entre medidas enteras, si bien es posible utilizar términos subjetivos, y en la utilización preferente de términos que indican ausencia de valoración objetiva fija y que se refieren a una estructura dicotómica con una conexión imprecisa o indeterminada entre las partes, (propia de un orden parcial con inversión en la región "negativa") en las comparaciones de medidas naturales relativas.

En definitiva, aunque se ha constatado que no se dominan en la misma medida los diferentes tópicos o contextos concretos que se han utilizado en el cuestionario, en el que coexisten situaciones familiares con una gran homogeneidad en las respuestas junto a situaciones heterogéneas en cuanto al conocimiento de sus características y modo de funcionamiento, se puede afirmar que los individuos de la muestra valoran de manera diferente los dos tipos de situaciones, delimitando, en un alto porcentaje, el campo de los números naturales relativos y el campo de los números enteros. Es por ello que podemos decir, por un lado, que los criterios empleados en la construcción del cuestionario han sido los correctos salvo pequeñas modificaciones puntuales y que el cuestionario ha constituido un instrumento idóneo para reflejar el tratamiento diferenciado a los dos tipos de situaciones que se proponen; por otro lado, los 
resultados sostienen la plausibilidad de la hipótesis empírica sometida a crítica, es decir: Individuos con estudios superiores al nivel correspondiente a 14 años de edad dan un tratamiento semántico diferenciado a los números naturales relativos y a los números enteros sobre la base de sus diferencias ordinales, cuando se presentan en situaciones elementales de comparación de medidas discretas

Pero esta afirmación es, además, sorprendente, si tenemos en cuenta que la instrucción matemática recibida por los sujetos de la muestra está sustentada en el supuesto de que la estructura entera es el único ámbito idóneo para el tratamiento directo y en las mismas condiciones de todas las situaciones planteadas en el cuestionario. Los resultados obtenidos contradicen dicho supuesto, quedando abierto el problema didáctico de cómo afrontar los desajustes detectados entre el pensamiento numérico natural relativo y el entero, o entre el pensamiento aritmético y el algebraico, y cómo abordar la transición de los naturales a los enteros y de la aritmética al álgebra.

Por último, es evidente la necesidad de estudios de replicación que corroboren los indicios encontrados, profundizando las tipologías de respuestas emitidas en todos los cuestionarios. Asímismo creemos necesario un estudio específico para profundizar sobre los motivos de las disparidades existentes con respecto a las situaciones irregulares o de respuesta no esperada que, aunque son minoría, se muestran como tales en todos los cuestionarios. 


\section{Referencias}

Bisquerra, R. (1989). Métodos de investigación educativa. Barcelona: CEAC.

Castro, E. (1995). Exploración de patrones numéricos mediante configuraciones puntuales. Estudio con escolares de primer ciclo de Secundaria (12-14 años). Tesis doctoral. Granada: Comares.

Clawson, C. (1994). The Mathematical Traveler. Exploring the grand History of Numbers. New York: Plenum Press.

Coquin-Viennot, D. (1985). Complexitè mathèmatique et ordre d'adquisition: une hierarchie de conceptions a propos des relatifs. Recherches en Didactique des Mathèmatiques, 6(2-3), pp. 133-192.

Cornejo, J. M. (1988). Técnicas de investigación social: el análisis de correspondencias. Barcelona: PPU.

Euler.L. (1984). Elements of Algebra, reimpresión de la quinta edición. Londres: Longman, Orme and Co., 1840. New York: Springer-Verlag.

Feferman, S. (1989). The Numbers Systems Foundations of Algebra and Analysis. New York: Chelsea Publishing Company.

Fernandez, A. (1995). Métodos para evaluar la investigación en Psicopedagogía. Madrid: Síntesis.

Gericke, H. (1996). Zur Gegschitche der negativen Zahlen. En: Dauben, J.; Folkerts, M.; Knobloch, E.; Wu $\square$ ing, H. (eds.). History of Mathematics. State of Art. (pp. 279-306). San Diego CA: Academic Press.

Glaeser, G. (1981). Epistémologie des nombres relatifs. Recherches en Didactique des Mathèmatiques, 2(3), 303-346.

González, J. L. (1990). Números enteros. Madrid: Síntesis.

González, J. L. (1995). El campo conceptual de los números naturales relativos. Tesis doctoral. Málaga: Servicio de Publicaciones de la Universidad de Málaga (formato microfichas).

González, J. L. (1997). Numbers and measurements in the additive conceptual field. En: Proceedings of the 21th Conference of the International Group for the Psychology of Mathematics Education (PME21). Lahti (Finland). Vol. 1, p. 280.

González, J. L. (1998). Números Naturales Relativos. Granada: Comares.

Maz, A. (2000). Los números negativos en textos de matemáticas españoles publicados en el siglo XIX Memoria de Tercer Ciclo. Departamento de Didáctica de la Matemática. 
Universidad de Granada.

Nesher, P., Greeno, J. G. y Riley, M. S. (1982). The development of semantic categories for Addition and Subtraction. Educational Studies in Mathematics, 13, 373-394.

Osgood, C.E., Suci, G.J. y Tannerbaum, P. H. (1957). The Measurement of Meaning. Illinois: University of Illinois Press. Urbana IL.

Ogawa, R.T. y Malen, B. (1991). Towards rigour in reviews of multivocal literature: Applying the exploratory case study method. Review of Educational Research, 61(3), pp. 265-286.

Pycior, H. M. (1981). George Peacock and the British origins of Symbolical Algebra. Historia Mathematica, 8, 23-45.

Rico, L. y Castro, E. (1995). Pensamiento Numérico en Educación Secundaria Obligatoria. En Aspectos didácticos de Matemáticas (pp.163-182). Zaragoza: ICE Universidad de Zaragoza.

Russell, B. (1973). Ciencia y Filosofía. Madrid: Aguilar.

Schubring, G. (1986). Ruptures dans le statut mathèmatique des nombres negatifs. Petit X, $12,5-32$.

Stegmüller, W. (1979). Teoría y experiencia. Barcelona: Ariel.

Summers, G. F. (1982). Medición de actitudes. México: Trillas.

Ulloa, P. (1706). Elementos matemáticos. Madrid: Antonio González Reyes.

Vergnaud, G. (1993). La teoría de los campos conceptuales. En: Sánchez, E.; Zubieta, G. (eds.). Lecturas en Didáctica de las Matemáticas. Escuela Francesa (pp. 88-117). México: Cinvestav-IPN. 


\section{Anexo 1. Cuestionario definitivo empleado en el estudio}

\section{(Pon una cruz en las casillas que creas conveniente)}

(Antes de contestar a cada pregunta, lee las opciones de la parte superior del cuadro)

Si quieres tachar una cruz que ya has círculo así: colo cado por equivocación, dibuja un

SON SIT UACIONES CONT RARIAS

QUE NORIIALMENTE SE VALORAN MEDIANTE EL PAR DE TERMINOS:

do acreedor/ un saldo deu dor

Cuando voy a comprar,

Faltar dinero / sobrar dinero

Cuando estoy jugando,

una gan ancia/ un a pérdida

Con relación a un a fecha dada

un día previo / un día después

En mi cuenta bancaria,

un ingreso / un reintegro

Una temperatu ra bajo cro /

una temperatura sobre cern

Con relación al nivel del mar

una altu ra p or encima / una p or debajo

En un ascensor

un descenso / un ascenso

Un año previo al nacimiento de Cristo

$$
\text { un año después }
$$

Una bajada / una subida

de una cantidad de escalones

$$
\text { En una persona, }
$$

un aumento / una disminución de peso

En la econo mía de mi familia, un balan-

ce conmás gastos que ingresos/viceversa

En una parada de autobús, una subida/

una bajada de un número de personas

En un edificio, un piso por debajo de la

plan ta baja / uno por encima

En el golf, una puntuación "sobre par" / una "bajo par"

En una cafetería, una salida / una en-

trada de un $n^{\circ}$ de personas

En una ciudad, un aumento / una dis-

minución de temperatura

Perd er u na cantidad de botones/encon-

trar una cantidad de botones

A la edad de una persona, añ adir un $n^{\circ}$

de años / quitar un $\mathrm{n}^{\circ}$ de años

Con respecto a la hora del lanzamiento,

un segundo previo/un segundo después

En un g lobo, un ascenso / un descenso de un $n^{\circ}$ de metros

Un índice que indica una bajada de la

bolsa / uno que indica una subida

En una cita, minutos que han pasado / minutos que faltan

Un descuen to sobre el precio de un

artículo / un recargo sobre dicho precio
PARA LAS QUE

NO EXISTE UNA

VALORACION

FJA

Y POR TANTO:

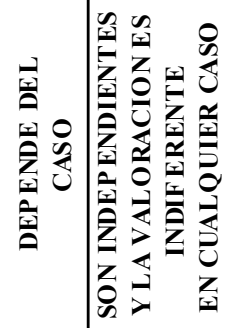


Anexo 2. Esquema del análisis de correspondencias

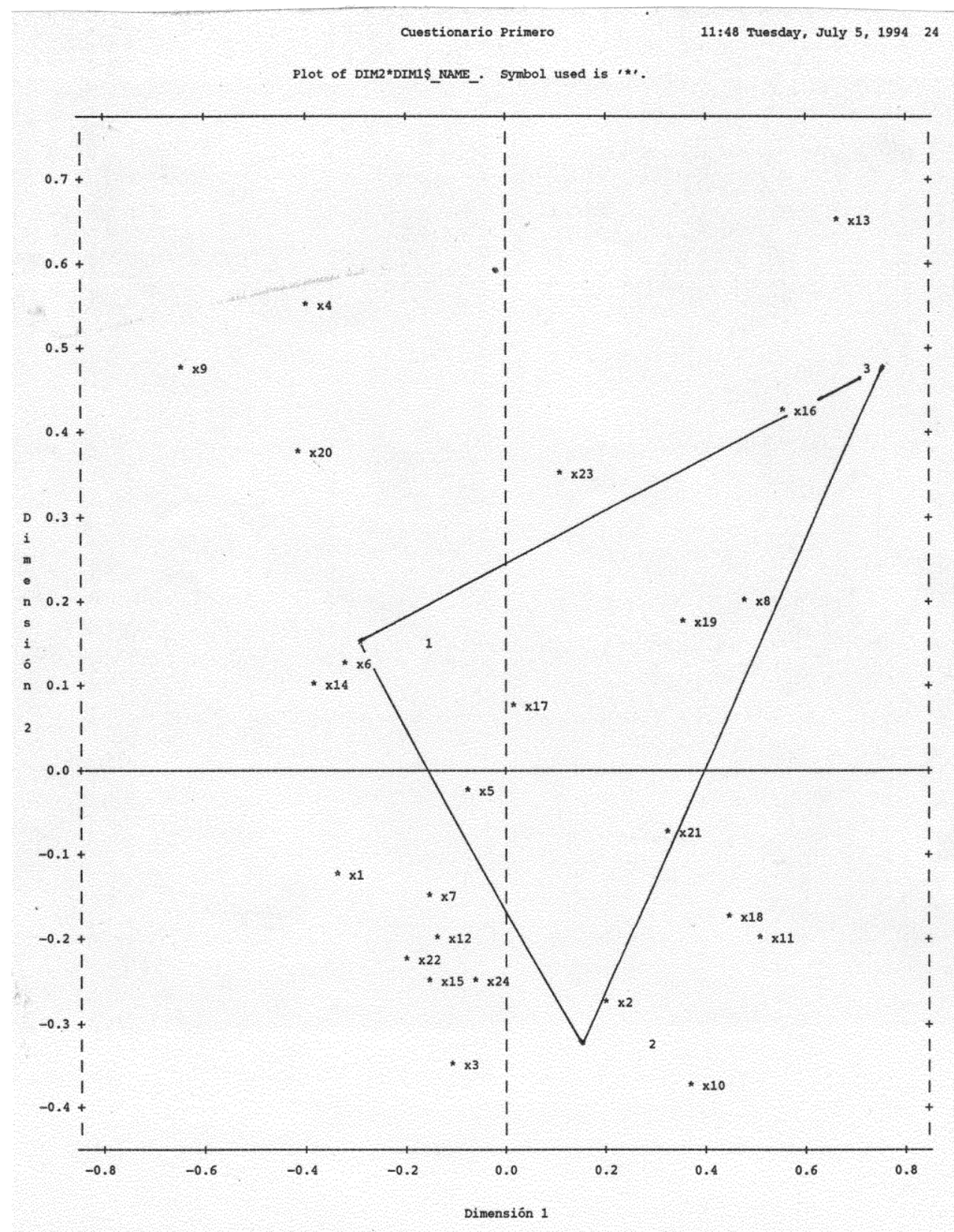

NOTE: 1 obs had missing values. 
José Luíz González et al

[Página en blanco por razones de paginación] 